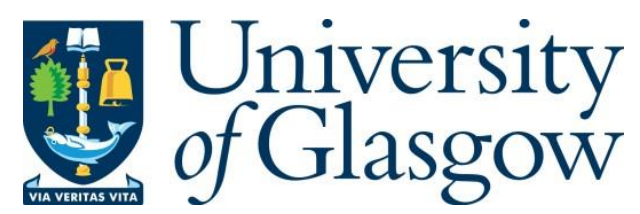

Chung, L. H. C., Birch, D. J. S., Vyshemirsky, V., Ryadnov, M. G. and Rolinski, O. J. (2019) Tracking insulin glycation in real time by time-resolved emission spectroscopy. Journal of Physical Chemistry B, 123(37), pp. 7812-7817.

There may be differences between this version and the published version. You are advised to consult the publisher's version if you wish to cite from it.

$\underline{\text { http://eprints.gla.ac.uk/196431/ }}$

Deposited on: 9 September 2021

Enlighten - Research publications by members of the University of Glasgow http://eprints.gla.ac.uk 


\title{
Tracking Insulin Glycation in Real Time by Time-Resolved Emission Spectroscopy
}

Li Hung C. Chung, ${ }^{1}$ David J. S. Birch, ${ }^{1}$ Vladislav Vyshemirsky, ${ }^{2}$ Maxim G. Ryadnov, ${ }^{3}$ and Olaf J. Rolinski $i^{*}$

\author{
${ }^{1}$ Photophysics Group, Centre for Molecular Nanometrology, Department of Physics, Scottish \\ Universities Physics Alliance, University of Strathclyde, 107 Rottenrow East, Glasgow G4 \\ $0 \mathrm{NG}, \mathrm{UK}$ \\ ${ }^{2}$ School of Mathematics and Statistics, University of Glasgow, Glasgow G12 8QQ, UK \\ ${ }^{3}$ National Physical Laboratory, Hampton Road, Teddington TW11 0LW, UK
}

\begin{abstract}
The application of time-resolved fluorescence sensing to the study of heterogenic biomolecular systems remains challenging due to the complexity of the resulting photophysics. Measuring the time-resolved emission spectra (TRES) can provide a more informative alternative to the modelling of the fluorescence decay that is currently employed. Here we demonstrate this approach by monitoring real-time changes in intrinsic insulin fluorescence by TRES as a straightforward probe to directly measure kinetics of insulin aggregation and glycation. Our findings hold promise for monitoring the storage of insulin and its application in the control of diabetes, and may support the development of more effective therapeutics against amyloidosis.
\end{abstract}

* Corresponding author: o.j.rolinski@strath.ac.uk 


\section{Introduction}

Protein aggregation has been intensively studied over the past decades due to its role in neurodegenerative diseases including Alzheimer's, Parkinson's and diabetes mellitus. ${ }^{1,2}$ The process results from the misfolding of otherwise normally functional soluble proteins, leading to the formation of amyloid fibrils and eventually insoluble amyloid deposits in the brain of patients.

Insulin is a hormonal protein which stabilizes blood glucose levels, when produced in the pancreas. It is stored as medicine in its most stable hexameric form. ${ }^{3}$ However, insulin aggregation still occurs in its stabilized form, which becomes problematic for long term storage. Insulin fibrils formed as the result of aggregation are found to be cytotoxic ${ }^{4}$ and cause localized amyloidosis at frequent injection sites. ${ }^{5}$ Moreover, insulin oligomers and fibrils cause an autoimmune response in serum from patients with Parkinson's disease. ${ }^{6}$ Despite insulin aggregation occurring under physiological conditions, a great deal of studies were conducted under non-physiological conditions such as acidic $\mathrm{pH}$ and high temperatures, which accelerate fibril formation. ${ }^{7-9}$

Insulin is associated with glycaemia but reducing sugars are known to interact and modify proteins through a non-enzymatic reaction known as glycation. Glycation occurs when the carbonyl group of the sugar and a free amino group (typically from arginine and lysine residues, or N-terminal amino group) forms a Schiff's base. ${ }^{10}$ Amadori products are produced when the Schiff base rearranges itself into a stable ketoamine. Dehydration reactions and further rearrangements generate advanced glycation end products (AGEs) and play a role in diabetes and cataracts. Glycated proteins are found in Lewy bodies ${ }^{11}$ and $\beta$-amyloid $(\mathrm{A} \beta)^{12}$ deposits which are related to Parkinson's disease and Alzheimer's disease, respectively. 
Therefore, it is thought that reducing sugars could induce protein aggregation and misfolding, and the formation of cross-links with protein which increases the stability of protein aggregates ${ }^{13}$. Glycated insulin is unable to transport glucose to cells and inhibits the regulation of blood glucose, therefore, reducing sugars play a key role in insulin resistance $e^{14,15}$.

The insulin monomer consists of two polypeptide chains, named A and B. Chain A has 21 amino acids and $\mathrm{B}$ has 30 amino acids, both chains are linked together by two disulphide bridges. Together they contain seven intrinsic fluorophores: four tyrosine (Tyr) and three phenylalanine (Phe) residues. However, the hexameric insulin form produced and stored in the pancreas consists of three dimers stabilized by zinc ions. Using mass spectrometry, glucose binding sites have been found Lys (B29) in chain B and in the N-terminus of both chains $\mathrm{A}$ and $\mathrm{B}^{16,17}$. From nuclear magnetic resonance nuclear Overhauser effect spectroscopy (NMR NOESY) experiments it was found that Val (B2) and Leu (B17) were the best residues for glucose interaction ${ }^{18}$ and molecular dynamics (MD) simulations showed that glucose had a large affinity towards the hydrophobic pocket between Val (B2) and Leu $(\mathrm{B} 17)^{19}$. It has been reported that free insulin in non-reducing conditions has the tendency to form oligomers, whereas oligomerization in glycated insulin is $1 \mathrm{ow}^{20}$, therefore glucose inhibits amyloid formation ${ }^{21}$. Although significant glycation occurs over a number of weeks ${ }^{22}$, insulin glycation is measured in non-physiological conditions spanning only over a few hours or days.

In our previous study ${ }^{23}$, we have explored the sensitivity of the intrinsic tyrosine fluorescence to study insulin alterations and recovered the kinetics of its aggregation with the use of timeresolved emission spectra (TRES). It has been found that hexameric insulin under physiological conditions undergoes conformational changes throughout 700 hours. This was further supported by similar assembly kinetics of a model fibrillar protein ${ }^{24}$ suggesting a 
generic mechanism and that the sufficient sensitivity of TERS to monitor protein stability for medicinal purposes.

In this present study, we demonstrate the medical application of this methodology and compare evolution of TRES of free insulin and glycated insulin. As there are no tryptophan (Trp) residues in insulin, the fluorescence kinetics are simplified. Understanding this kinetics is likely to provide insights into the mechanism of insulin glycation which, in turn, may enable the development of better therapeutics to halt or circumvent fibril formation.

\section{Methods}

Bovine insulin, D-(+)-Glucose, and phosphate-buffered saline pH 7.4 (PBS) from SigmaAldrich were used without further purification. Two $50 \mu \mathrm{M}$ bovine insulin samples were prepared in PBS buffer $(0.01 \mathrm{M})$. A stock solution of D-(+)-Glucose in distilled water was added to one bovine insulin sample and the final concentration of glucose was $50 \mathrm{mM}$. The samples were sonicated, stored, and measured at $22^{\circ} \mathrm{C}$. The absorption and fluorescence steady state spectra were measured with the use of Lambda $25 \mathrm{UV}$-Vis spectrometer (PerkinElmer) and Fluorolog (Horiba Scientific), respectively. A series of time-resolved fluorescence decays were measured on a DeltaFlex fluorescence lifetime system (Horiba Jobin Yvon IBH Ltd, Glasgow) with the use of time correlated single photon counting (TCSPC) technique. To excite intrinsic tyrosine fluorescence within the sample, a $279 \mathrm{~nm}$ NanoLED was used ${ }^{25}$. In order to retrieve sufficient amount of data for TRES calculations, the fluorescence decay detection wavelengths $\lambda$ ranged from $290-350 \mathrm{~nm}$ at $5 \mathrm{~nm}$ intervals. Polarization effects are limited within the fluorescence decay by setting the polarizer in the detection channel at the magic angle $\left(54.7^{\circ}\right)$ in respect to vertically-oriented polarizer in the 
excitation channel. The series of fluorescence decays for a range of wavelengths were fitted to a 3-exponential function.

$$
I_{\lambda}(t)=\sum_{i=1}^{3} \alpha_{i}(\lambda) \exp \left(\frac{-t}{\tau_{i}(\lambda)}\right)
$$

where $\tau_{i}(\lambda)$ and $\alpha_{i}(\lambda)$ are the fluorescent lifetimes and pre-exponential factors with respect to each detection wavelength, respectively. The goodness of fit criteria were examined with the use of the nonlinear least squares method. The weighted residuals and $\chi^{2}$ indicated that a 3-exponential function described the fluorescence decay adequately.

The recovered parameters $\tau_{i}(\lambda)$ and $\alpha_{i}(\lambda)$ were then used to generate TRES $I_{t}(\lambda)$ :

$$
I_{t}(\lambda)=\sum_{i=1}^{3} \frac{I_{\lambda}(t) S(\lambda)}{\sum_{i} \alpha_{i}(\lambda) \tau_{i}(\lambda)}
$$

where $S(\lambda)$ is the steady state spectrum of the sample. Experimental TRES have been converted to the wavenumber scale according to

$$
I_{t}(v)=\lambda^{2} I_{t}(\lambda)
$$

and modelled in terms of the multiple normalised Toptygin-type distributions ${ }^{26}$ which represent the spectral shapes of individual fluorescence components within the superimposed TRES.

$$
F(v)=\sum_{i=1}^{N} \frac{C_{i}}{\sqrt{2 \pi} v_{i} \sigma_{i}\left(v_{i}^{2}+3 \sigma_{i}^{2}\right)} v^{3} \exp \left(\frac{-\left(v-v_{i}\right)^{2}}{2 \sigma_{i}^{2}}\right)
$$

where $C_{i}, v_{i}$ and $\sigma_{i}$ is the fluorescence contribution, peak position, and half-width of the distribution of each component, respectively. The expression $\left(\sqrt{2 \pi} v_{i} \sigma_{i}\left(v_{i}^{2}+3 \sigma_{i}^{2}\right)\right)^{-1}$ is the normalised factor for each spectrum component. The Toptygin-type model (4) parameters 
were recovered by fitting the distributions $v^{-3} F(v)$ to the experimental TRES expressions of $v^{-3} I_{t}(v)$

\section{Results and discussion}

The steady state emission spectra for free insulin (figure 1A) shows that from 0.5 to 310 hours of incubation time the fluorescence intensity decreases but the shape of the spectra is maintained. The decrease in tyrosine fluorescence intensity was also observed by Bekard et al. ${ }^{27}$ and there was an increase in the 1-anilinonaphthalene-8-sul- phonic acid (ANS) signal measured in a 22 hour time range. Tyrosine residues (A14), (B16) and (B26) are located on hydrophobic surfaces within insulin ${ }^{28}$ and ANS is a probe commonly used to bind in hydrophobic regions. Therefore, the decrease in intensity may be due to the conformational changes which expose tyrosine residues to quenchers in the solution. After about 310 hours, the spectra experience a further decrease and broaden quite significantly. The normalised spectra (figure 1B) demonstrate large broadening with a new peak emerging around $330 \mathrm{~nm}$. These effects are likely to be a result of formation of aggregates in a distribution of conformations which fluorescent at longer wavelengths.
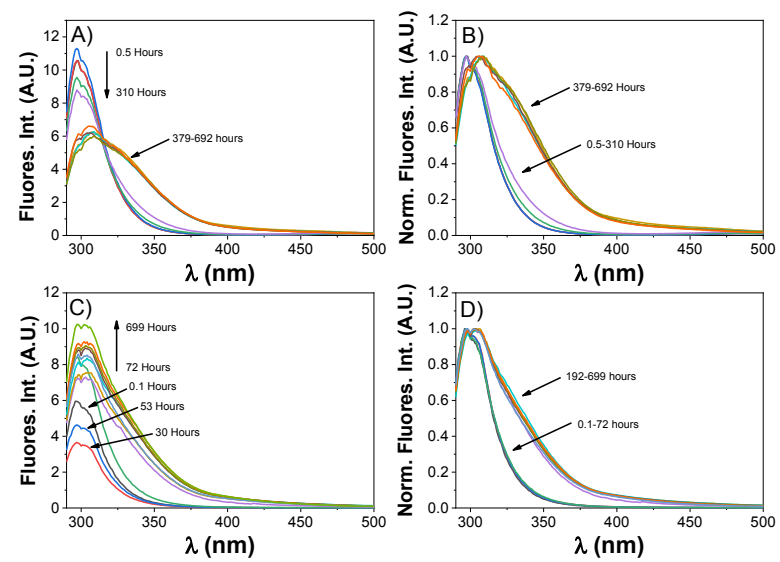

Figure 1: Fluorescence intensity of free insulin A) at $0.5,26,44,196,310$, $379,459,528,605,692$ hours of preparation and insulin with glucose C) measured at $0.1,30,53,72,192,318,384,479,527,577,655$ and 699 hours after preparation. The corresponding normalised intensities are shown in B) and D) respectively. 
In case of when insulin was incubated with glucose the fluorescence intensity (shown in figure 1C) decreased at a time range of 0.1 to 30 hours and then starts to increase. For the first 30 hours the trend appeared to be similar to that of free insulin but it started at a lower level, which may be due to glucose quenching the overall tyrosine fluorescence. At later times glucose inhibits quenching of tyrosine which may be caused by glucose acting as a shield around insulin and tyrosine is no longer exposed to the water, which in turn, increases tyrosine fluorescence. The normalised spectra shown on figure 1D also shows that the spectra starts to broaden after 192 hours and there is a second peak around $330 \mathrm{~nm}$, which is not as prominent as in free insulin.

The readings of the fluorescence peak intensities were taken at $298 \mathrm{~nm}$ for both free insulin and insulin with glucose and were plotted on figure $2 \mathrm{~A}$. The total fluorescence intensities were also calculated by taking the integral of the fluorescence spectrum of each measured sample (figure 2B). The peak intensity for free insulin decreases but the integrated fluorescence intensity remains constant. This is consistent with formation of aggregates of different sizes, each showing varied peak positions on the spectrum.
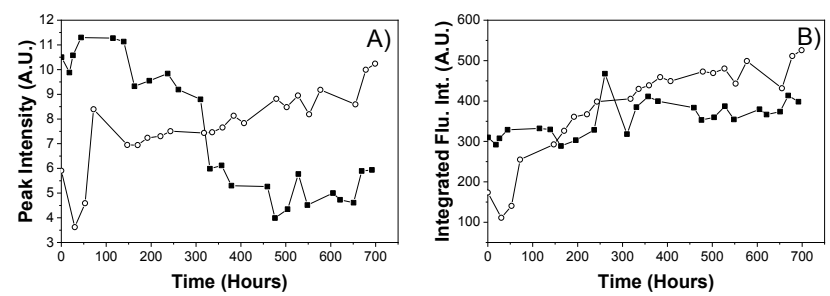

Figure 2: A) Peak fluorescence intensity at $298 \mathrm{~nm}$ and B) integrated fluorescence spectra taken from $50 \mu \mathrm{M}$ insulin (ם) in PBS buffer and $50 \mu \mathrm{M}$ insulin with $50 \mathrm{mM}$ (O) glucose in PBS buffer. 
For glycated insulin, both the peak intensity and total number of photons emitted increase, which, in turn, suggests not only formation of aggregates (broadening the spectra), but also sugar molecules separating Tyr from the solvent (which increases quantum yield, thus also fluorescence intensity).

The fluorescence decays for free and glycated insulin were measured at several times over the period of about 700 hours of incubation. The emission wavelength detection ranged from 290 $-350 \mathrm{~nm}$ in increments of $5 \mathrm{~nm}$. It can be observed (Fig.3) that increasing incubation time of both samples results in longer lifetimes and the spread of the decays are more significant in the free insulin sample. This may indicate that the glucose-insulin complexation makes tyrosine local environments less heterogenic.
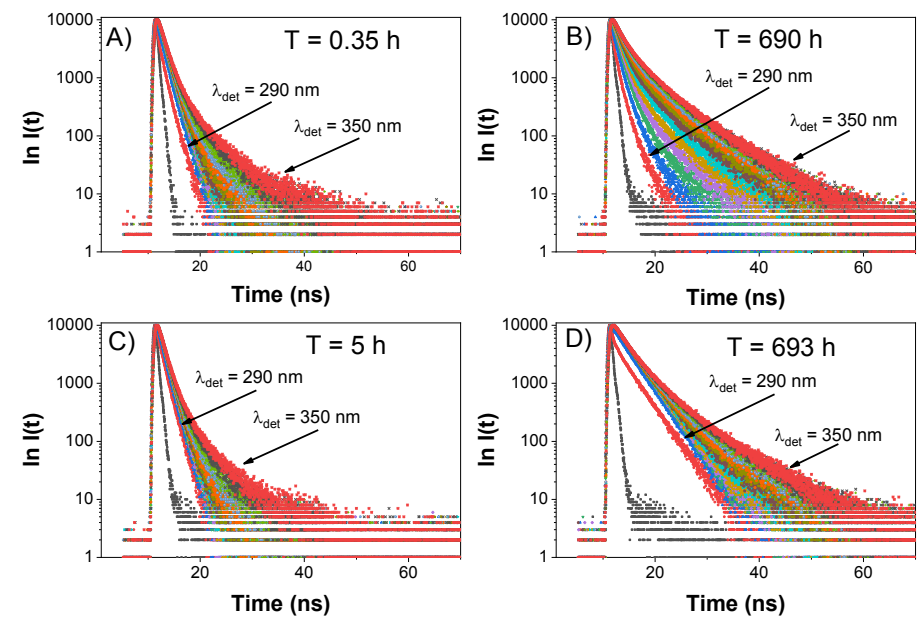

Figure 3: Raw fluorescence decays of free insulin measured at A) 0.35 and B) 690 hours and insulin with glucose measured at C) 5 and D) 693 hours after sample preparation.

Figure 4 shows examples of TRES of the free insulin and glycated insulin samples after 0.35 , 194 and 690 hours of incubation. At 0.35 hours (Fig.4.A and B), the fluorescence intensity of free insulin is lower as compared with the insulin with glucose, but the normalised TRES plots show similar trends. The increased intensity in the sample with glucose is consistent with the steady-state data and may be explained by glucose shielding insulin from water 
molecules that would normally quench tyrosine fluorescence. After 194 hours (Fig.4.C and D), glucose influence on tyrosine fluorescence is substantially different: the intensity, as compared to free insulin, decreases faster at higher wavenumbers and slower at smaller wavenumbers. This may be explained in terms of the variety of possible complexes between
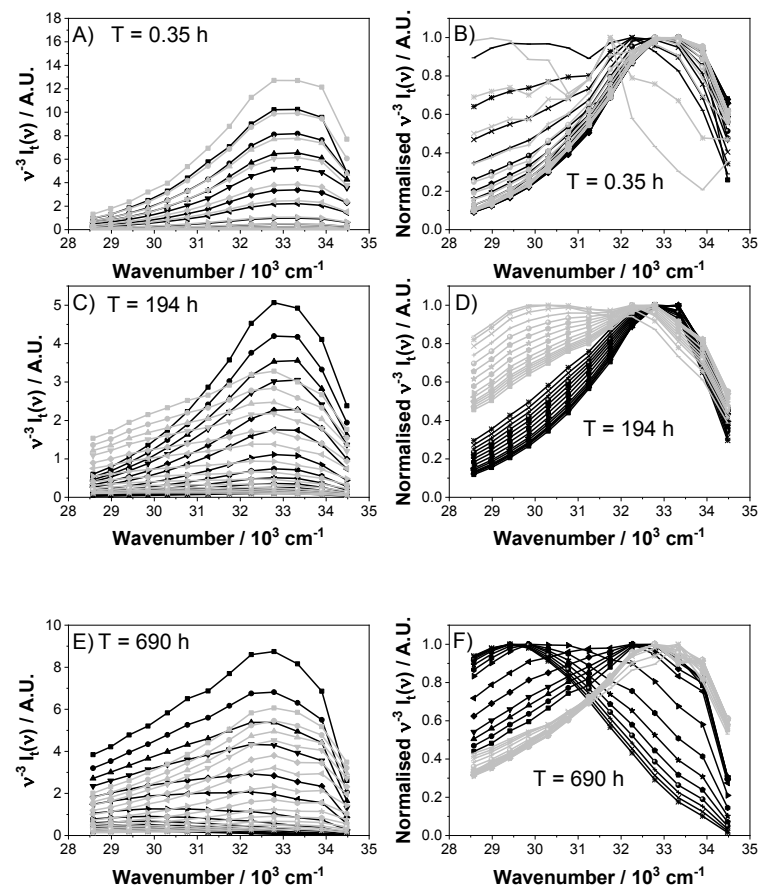

Figure 4: TRES of free insulin (black curves) and insulin with glucose (grey curves). The left hand panel demonstrates the evolution of time $0,0.25,0.5,0.75,1$, $1.5,2,3,4,5,6,7,8,9$, and $10 \mathrm{~ns}$ after excitation. Samples were measured at times 0.35 (A), 194 (C), and 690 (E) hours. The right hand panel illustrates normalised TRES.

non-glycated, non-glycated/glycated, and glycated only insulin molecules, which results in much broader range of local environments for tyrosine, thus broadening the fluorescence spectrum. During the initial $\sim 300$ hours we can observe the two dominating bands in the TRES, one at $\sim 32500 \mathrm{~cm}^{-1}$, which is the tyrosine fluorescence in glycated insulin, slightly shifted towards lower energies (as compared to free insulin) due to sugar binding, and the other one around $\sim 30000 \mathrm{~cm}^{-1}$, which is likely to be fluorescence of the insulin glycation 
products. Their intensity decays are slower than these of the non-complexed molecules, because the TRES peak at $30000 \mathrm{~cm}^{-1}$ decreases slower than the one at $32500 \mathrm{~cm}^{-1}$.

For the glycated sample incubated for longer than 350 hours (see Supporting Information), the fluorescence peak of the complexes shifts towards higher energies and the rate of its decay becomes faster and at times 500-700 hours the TRES almost does not change its shape. At the moment we explain this by glycated insulin aggregates binding together to form fibrils/AGEs, which are more rigid than smaller aggregates. This results in limited orientational freedom for tyrosines (more stable TRES) and quenching by tightly packed complexes (shorter lifetimes).

For quantitative interpretation of the TRES results, the 2-component spectrum model (Eqtn 4) was fitted to the TRES spectra of both samples and its parameters were recovered. The positions of spectral peaks $v_{i}(t)$ are plotted in figure 5 .
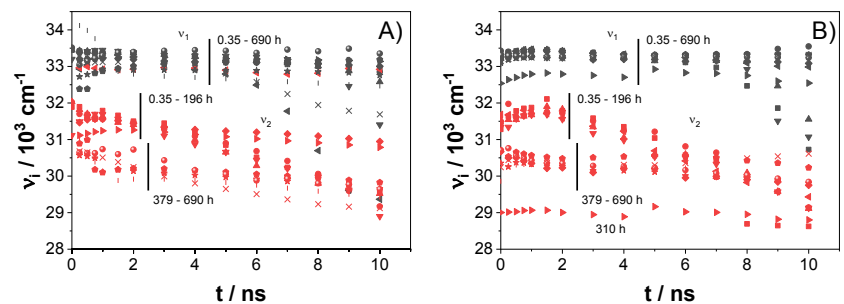

Figure 5: Gaussian models were used to fit TRES spectra for A) free insulin and B) insulin with glucose and the peak positions $v_{i}$ were extracted and plotted for $0.33(\boldsymbol{\bullet}), 22(\bullet), 42$ $(\varangle), 68(\mathbf{\Delta}), 93(\boldsymbol{\nabla}), 196(\diamond), 310(\triangleright) 379(\star), 458(\bullet)$, $528(\bullet), 605(\mid)$, and $690(X)$ hours. The components 1 and 2 are represented with grey and red symbols, respectively.

The higher energy peaks $v_{l}(t)$ occur for both samples around $33,000 \mathrm{~cm}^{-1}$ and remain stable throughout the measured 700 hours. The changes in the second peaks positions $v_{2}(t)$ depend substantially on the incubation times of the samples. During the first 200 hours $v_{2}(t)$ for both samples decrease from $\sim 32,000 \mathrm{~cm}^{-1}$ to $\sim 30,000 \mathrm{~cm}^{-1}$ during $10 \mathrm{~ns}$ after excitation, however 
there is a substantial difference between the free and glycated insulin. While the $v_{2}(t)$ in a free insulin sample has a decreasing trend in all stages of aging, $v_{2}(t)$ in the glycated sample seems to increase over the first two nanoseconds after excitation. We think this is due to the presence of a third fluorescent glycation product not appearing in a free insulin sample. Its presence increases the complexity of the fluorescence spectrum and the two-component model is no longer adequate, thus the apparent increase in $v_{2}(t)$ in the way the model accommodates the additional fluorescence.

In older samples, aged 379 to 690 hours of incubation, $v_{2}$ values start at lower level of $\sim 30,500 \mathrm{~cm}^{-1}$ which is probably caused by tighter aggregation of insulin proteins. The free insulin sample (red curves) still show a red-shift of $v_{2}$ to about $29500 \mathrm{~cm}^{-1}$, while the glycated sample (green curves) shows only slight shifts, confirming formation of more rigid structures.

\section{Conclusions}

To summarise, we suggest that observed modifications of TRES offer the opportunity to distinguish between different forms of protein glycation, thus offering systematic studies of glycation induced by variety of sugars, environmental conditions, anti-glycation factors, etc. However, this aim cannot be achieved by fitting experimental TRES to the more complex models of the spectrum, as this approach is already the subject of several assumptions (a 3exponential representation of the individual fluorescence intensity decays, Toptygin-type profile of the spectrum). Instead, the experimental TRES could be considered as an approximation and used to propose a selection of alternative models of the kinetics, for which the relevant fluorescence intensity decays $I_{\lambda}(t)$ will be calculated numerically. Confirmation of the specific type of glycation would be achieved of the basis of finding the best-fitting 
kinetic model and the values of its parameters. Our preliminary attempts to fit the numerical solutions of the kinetic models to the experimental decay data demonstrate full feasibility of this approach.

\section{Associated content}

Supporting Information: TRES of free insulin and insulin with glucose over $\sim 700 \mathrm{~h}$, parameters of Gaussian models fitted to TRES.

Author information:

*E-mail: o.j.rolinski@strath.ac.uk

\section{Acknowledgements}

LHCC acknowledges the support of a NPL-Strathclyde PhD case award.

\section{References}

(1) Knowles, T. P. J.; Vendruscolo, M.; Dobson, C. M. The Amyloid State and Its Association with Protein Misfolding Diseases. Nat. Rev. Mol. Cell Biol. 2014, 15, 384-396. https://doi.org/10.1038/nrm3810.

(2) Eisenberg, D.; Jucker, M. The Amyloid State of Proteins in Human Diseases. Cell 2012, 148, 1188-1203.

(3) Noormagi, A.; Gavrilova, J.; Smirnova, J.; Tougu, V.; Paulumaa, P. Zn ( II ) Ions CoSecreted with Insulin Suppress Inherent Amyloidogenic Properties of Monomeric Insulin. Biochem. J. 2010, 430, 511-518. https://doi.org/10.1042/BJ20100627.

(4) Zako, T.; Sakono, M.; Hashimoto, N.; Ihara, M.; Maeda, M. Bovine Insulin Filaments Induced by Reducing Disulfide Bonds Show a Different Morphology, Secondary Structure, and Cell Toxicity from Intact Insulin Amyloid Fibrils. Biophys. J. 2009, 96 (8), 3331-3340. https://doi.org/10.1016/j.bpj.2008.12.3957.

(5) Dische, F. E.; Wernstedt, C.; Westermark, G. T.; Westermark, P.; Pepys, M. B.; Rennie, J. A.; Gilbey, S. G.; Watkins, P. J. Insulin as an Amyloid-Fibril Protein at Sites of Repeated Insulin Injections in a Diabetic Patient. Diabetologia 1988, 31, 158161.

(6) Wilhelm, K. R.; Yanamandra, K.; Gruden, M. A.; Zamotin, V.; Malisauskas, M.; Casaite, V.; Darinskas, A.; Forsgren, L.; Morozova-Roche, L. A. Immune Reactivity towards Insulin, Its Amyloid and Protein S100B in Blood Sera of Parkinson's Disease Patients. Eur. J. Neurol. 2007, 14 (3), 327-334. https://doi.org/10.1111/j.1468- 


\subsubsection{7.x}

(7) Ahmad, A.; Uversky, V. N.; Hong, D.; Fink, A. L. Early in the Fibrillation of Monomeric Insulin. J. Biol. Chem. 2005, 280 (52), 42669-42675.

https://doi.org/10.1074/jbc.M504298200.

(8) Nielsen, L.; Khurana, R.; Coats, A.; Frokjaer, S.; Brange, J.; Vyas, S.; Uversky, V. N.; Fink, A. L. Effect of Environmental Factors on the Kinetics of Insulin Fibril Formation: Elucidation of the Molecular Mechanism. Biochemistry 2001, 40, 60366046. https://doi.org/10.1021/bi002555c.

(9) Hua, Q. X.; Weiss, M. A. Mechanism of Insulin Fibrillation: The Structure of Insulin under Amyloidogenic Conditions Resembles a Protein-Folding Intermediate. J. Biol. Chem. 2004, 279, 21449-21460. https://doi.org/10.1074/jbc.M314141200.

(10) Iannuzzi, C.; Irace, G.; Sirangelo, I. Differential Effects of Glycation on Protein Aggregation and Amyloid Formation. Front. Mol. Biosci. 2014, 1, 1-8. https://doi.org/10.3389/fmolb.2014.00009.

(11) Münch, G.; Lüth, H. J.; Wong, A.; Arendt, T.; Hirsch, E.; Ravid, R.; Riederer, P. Crosslinking of Alpha-Synuclein by Advanced Glycation Endproducts--an Early Pathophysiological Step in Lewy Body Formation? J. Chem. Neuroanat. 2000, 20, 253-257. https://doi.org/https://doi.org/10.1016/S0891-0618(00)00096-X.

(12) Sasaki, N.; Fukatsu, R.; Tsuzuki, K.; Koike, T.; Wakayama, I.; Yanagihara, R.; Garruto, R. Advanced Glycation End Products in Alzheimer's Disease and Other Neurodegenerative Diseases. Am. J. Pathol. 1998, 153, 1149-1155. https://doi.org/10.1016/S0002-9440(10)65659-3.

(13) Iannuzzi, C.; Irace, G.; Sirangelo, I. Role of Glycation in Amyloid : Effect on the Aggregation Process and Cytotoxicity. In Exploring New Findings on Amyloidosis; Fernandez-Escamilla, A. M., Ed.; InTech: Rijeka, 2016; pp 167-185. https://doi.org/10.5772/62995.

(14) Abdel-Wahab, Y. H. A.; O’Harte, F. P. M.; Barnett, C. R.; Flatt, P. R. Characterization of Insulin Glycation in Insulin-Secreting Cells Maintained in Tissue Culture. $J$.

Endocrinol. 1997, 152, 59-67. https://doi.org/Characterization of insulin glycation in maintained in tissue culture.

(15) Abdel-Wahab, Y. H. A.; O’Harte, F. P. M.; Ratcliff, H.; McClenaghan, N. H.; Barnett, C. R.; Flatt, P. R. Glycation of Insulin in the Islets of Langerhans of Normal and Diabetic Animals. Diabetes 1996, 45, 1489-1496. https://doi.org/10.1007/s005920050086.

(16) Guedes, S.; Vitorino, R.; Domingues, M. R. M.; Amado, F.; Domingues, P. Mass Spectrometry Characterization of the Glycation Sites of Bovine Insulin by Tandem Mass Spectrometry. J Am Soc Mass Spectrom 2009, 20, 1319-1326. https://doi.org/10.1016/j.jasms.2009.03.004.

(17) O'Harte, F. P. M.; Hojrup, H.; Barnett, C. R.; Flatt, P. R. Identification of the Site of Glycation of Human Insulin. Peptides 1996, 17, 1323-1330. https://doi.org/https://doi.org/10.1016/S0196-9781(96)00231-8. 
(18) Falconi, M.; Bozzi, M.; Paci, M.; Raudino, A.; Purrello, R.; Cambria, A.; Sette, M.; Cambria, M. T. Spectroscopic and Molecular Dynamics Simulation Studies of the Interaction of Insulin with Glucose. Int. J. Biol. Macromol. 2001, 29, 161-168. https://doi.org/10.1016/S0141-8130(01)00157-X.

(19) Zoete, V.; Meuwly, M.; Karplus, M. Investigation of Glucose Binding Sites on Insulin. PROTEINS Struct. Funct. Bioinforma. 2004, 55, 568-581. https://doi.org/10.1002/prot.20071.

(20) Alavi, P.; Yousefi, R.; Amirghofran, S. Structural Analysis and Aggregation Propensity of Reduced and Nonreduced Glycated Insulin Adducts. Appl Biochem Biotechnol 2013, 170, 623-638. https://doi.org/10.1007/s12010-013-0207-1.

(21) Barzegar, A.; Panahi, Y.; Pouladi, N. Reduction in Aggregation of Insulin through Conformational Stabilization by Glucose. Asian J. Chem. 2007, 19, 1749-1756.

(22) Singh, R.; Barden, A.; Mori, T.; Beilin, L. Advanced Glycation End-Products: A Review. Diabetologia 2001, 44, 129-146. https://doi.org/10.1007/s001250051591.

(23) Chung, L. H. C.; Birch, D. J. S.; Vyshemirsky, V.; Ryadnov, M. G.; Rolinski, O. J. Insulin Aggregation Tracked by Its Intrinsic TRES. Appl. Phys. Lett. 2017, 111, 263701. https://doi.org/https://doi.org/10.1063/1.5008477.

(24) Chung, L. H. C.; Birch, D. J. S.; Vyshemirsky, V.; Bella, A.; Ryadnov, M. G.; Rolinski, O. J. Protein Fibrillogenesis Model Tracked by Its Intrinsic Time-Resolved Emission Spectra. Methods Appl. Fluoresc. 2019, 7, 035003.

(25) McGuiness, C. D.; Sagoo, K.; McLoskey, D.; Birch, D. J. S. A New Sub-Nanosecond LED at $280 \mathrm{Nm}$ : Application to Protein Fluorescence. Meas. Sci. Technol. 2004, 15, 19-22. https://doi.org/10.1088/0957-0233/15/11/L02.

(26) Toptygin, D.; Brand, L. Spectrally- and Time-Resolved Fluorescence Emission of Indole during Solvent Relaxation: A Quantitative Model. Chem. Phys. Lett. 2000, 322, 496-502. https://doi.org/10.1016/S0009-2614(00)00460-7.

(27) Bekard, I. B.; Dunstan, D. E. Tyrosine Autofluorescence as a Measure of Bovine Insulin Fibrillation. Biophysj 2009, 97, 2521-2531.

https://doi.org/10.1016/j.bpj.2009.07.064.

(28) Strickland, E. H.; Mercola, D. Near-Ultraviolet Tyrosyl Circular Dichroism. Biochemistry 1976, 15 (17), 3875-3884.

\section{TOC GRAPHICS}




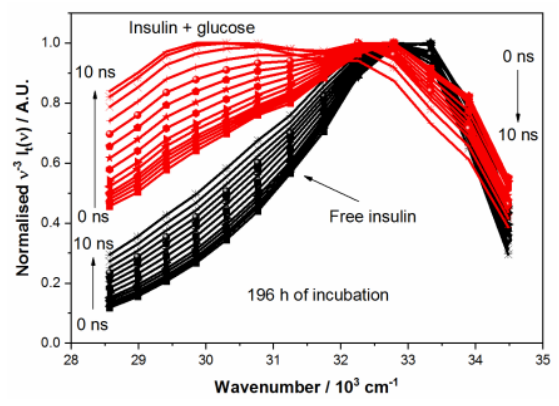




\section{Tracking Insulin Glycation in Real Time by Time-Resolved Emission Spectroscopy}

Li Hung C. Chung, ${ }^{1}$ David J. S. Birch, ${ }^{1}$ Vladislav Vyshemirsky, ${ }^{2}$ Maxim G. Ryadnov, ${ }^{3}$ and Olaf J. Rolinski $^{1^{*}}$

${ }^{1}$ Photophysics Group, Centre for Molecular Nanometrology, Department of Physics, Scottish Universities Physics Alliance, University of Strathclyde, 107 Rottenrow East, Glasgow G4 $0 \mathrm{NG}, \mathrm{UK}$

${ }^{2}$ School of Mathematics and Statistics, University of Glasgow, Glasgow G12 8QQ, UK

${ }^{3}$ National Physical Laboratory, Hampton Road, Teddington TW11 0LW, UK

\section{Supporting Information}
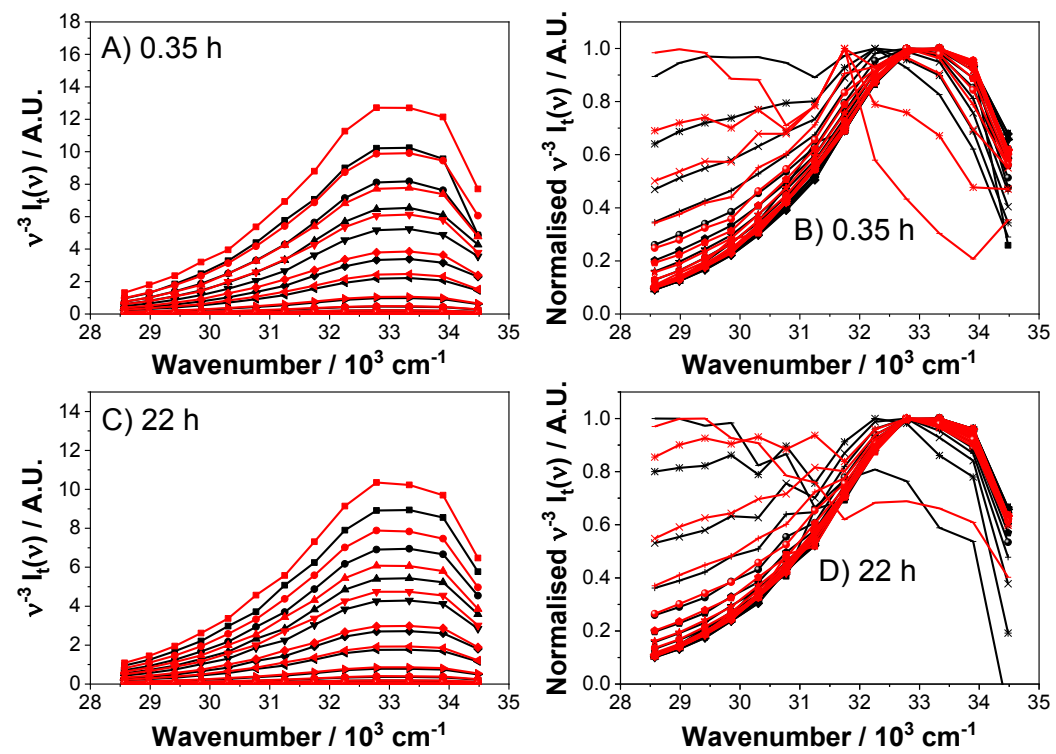

Figure S1: TRES of free insulin (black curves) and insulin with glucose (red curves). The left hand panel demonstrates the evolution of time $0,0.25,0.5,0.75,1,1.5,2,3,4,5,6,7,8$, 9, and $10 \mathrm{~ns}$ after excitation. Samples were measured at times A) 0.35, C) 22, E) 42, G) 68, I) 93, K) 196, M) 310, O) 379, Q) 458, S) 528, U) 605 and W) 690 hours. The right hand panel illustrates normalised TRES.

* Corresponding author: o.j.rolinski@strath.ac.uk 

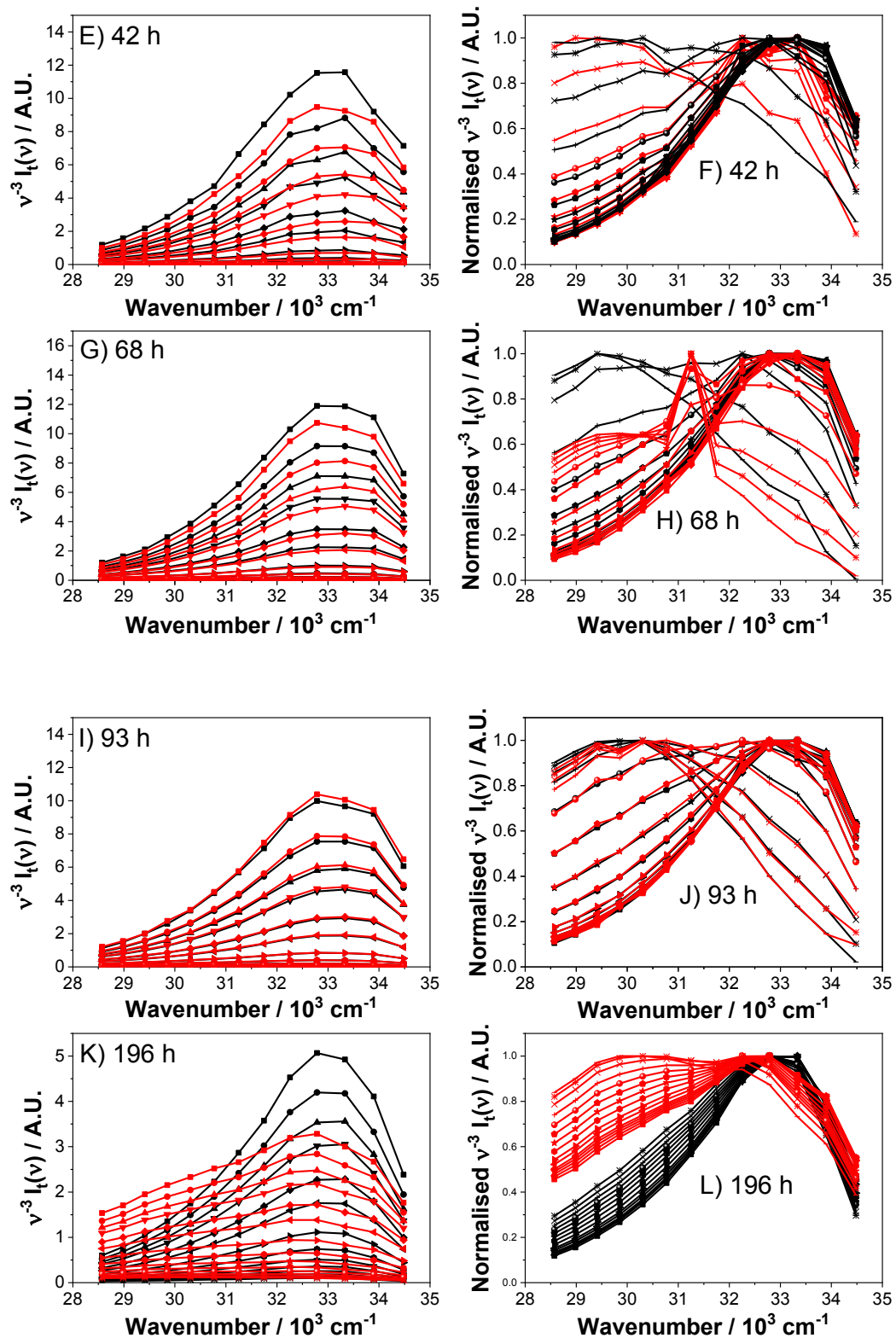

Figure S1-cont'd: TRES of free insulin (black curves) and insulin with glucose (red curves). The left hand panel demonstrates the evolution of time $0,0.25,0.5,0.75,1,1.5,2,3,4,5,6,7,8,9$, and $10 \mathrm{~ns}$ after excitation. Samples were measured at times A) 0.35, C) 22, E) 42, G) 68, I) 93, K) 196, M) 310, O) 379, Q) 458 , S) 528, U) 605 and W) 690 hours. The right hand panel illustrates normalised TRES. 

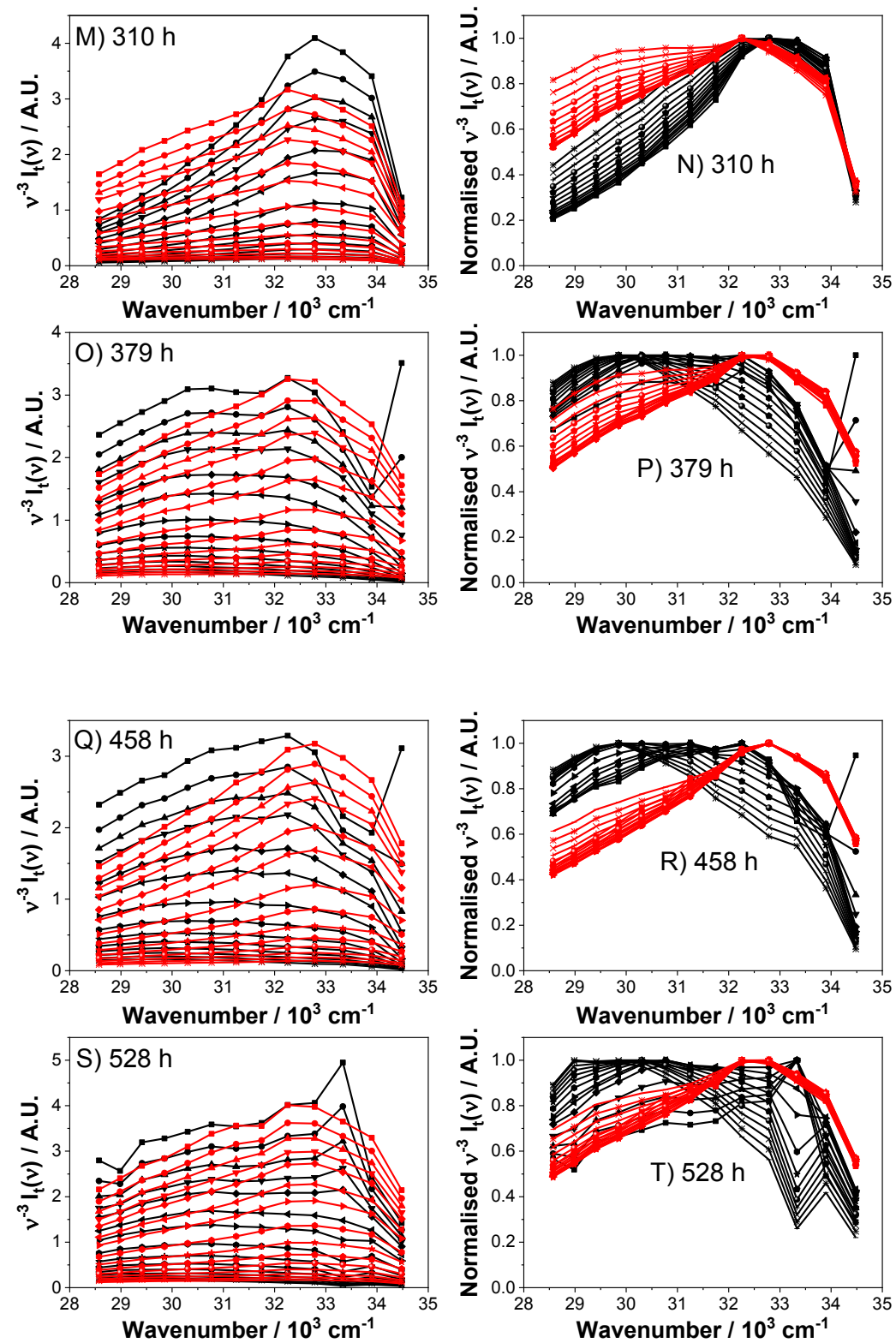

Figure S1-cont'd: TRES of free insulin (black curves) and insulin with glucose (red curves). The left hand panel demonstrates the evolution of time $0,0.25,0.5,0.75,1,1.5,2,3,4,5,6,7,8,9$, and $10 \mathrm{~ns}$ after excitation. Samples were measured at times A) 0.35, C) 22, E) 42, G) 68, I) 93, K) 196, M) 310, O) 379, Q) 458, S) 528, U) 605 and W) 690 hours. The right hand panel illustrates normalised TRES. 

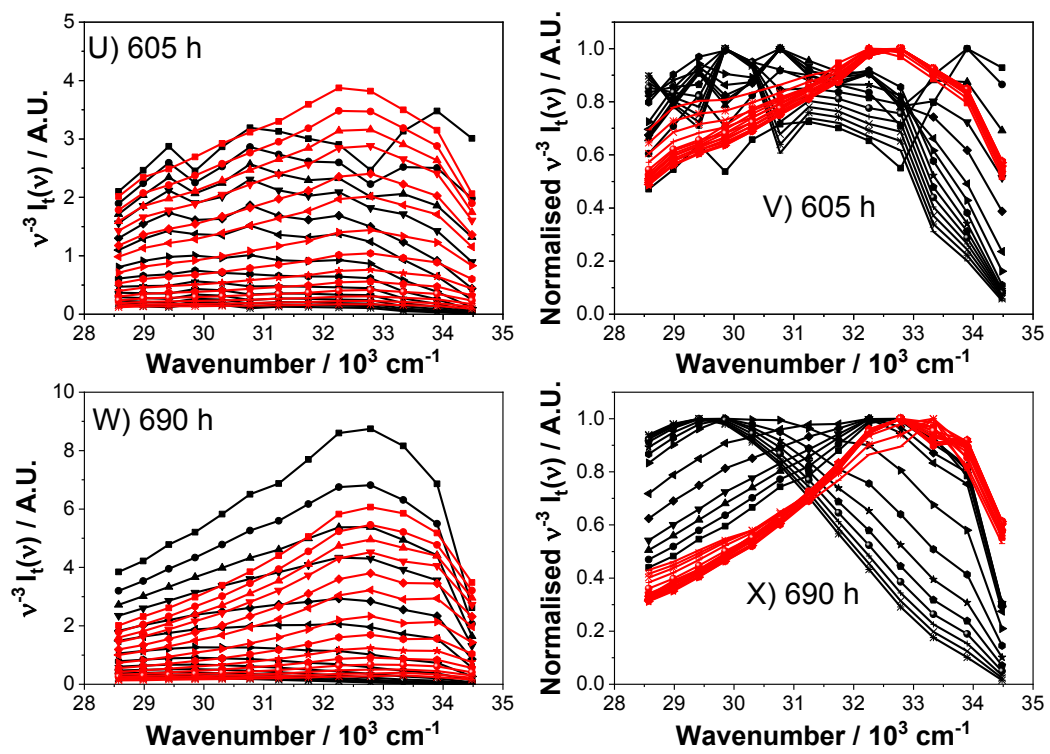

Figure S1-cont'd: TRES of free insulin (black curves) and insulin with glucose (red curves). The left hand panel demonstrates the evolution of time $0,0.25,0.5,0.75,1,1.5,2,3,4,5,6,7,8,9$, and $10 \mathrm{~ns}$ after excitation. Samples were measured at times A) 0.35, C) 22, E) 42, G) 68, I) 93, K) 196, M) 310, O) 379, Q) 458, S) 528, U) 605 and W) 690 hours. The right hand panel illustrates normalised TRES. 


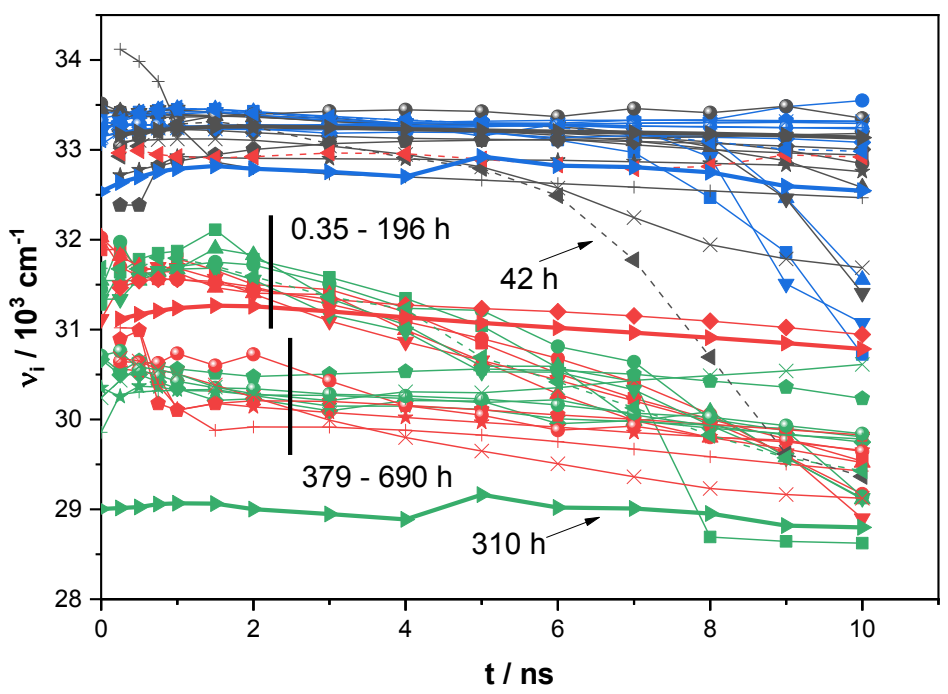

Figure S2: Gaussian models were used to fit TRES spectra for free insulin and insulin with glucose and the peak positions $\boldsymbol{v}_{\boldsymbol{i}}$ were extracted and plotted for $0.33(\boldsymbol{\bullet}), 22(\bullet), 42(\boldsymbol{\bullet}), 68(\boldsymbol{\Delta}), 93(\boldsymbol{\nabla})$, $196(\diamond), 310(\diamond) 379(\star), 458(\bullet), 528(\bullet), 605(\mid)$, and $690(\times)$ hours. Component 1 and 2 for free insulin and insulin with glucose are represented with grey, red, blue, and green, respectively.

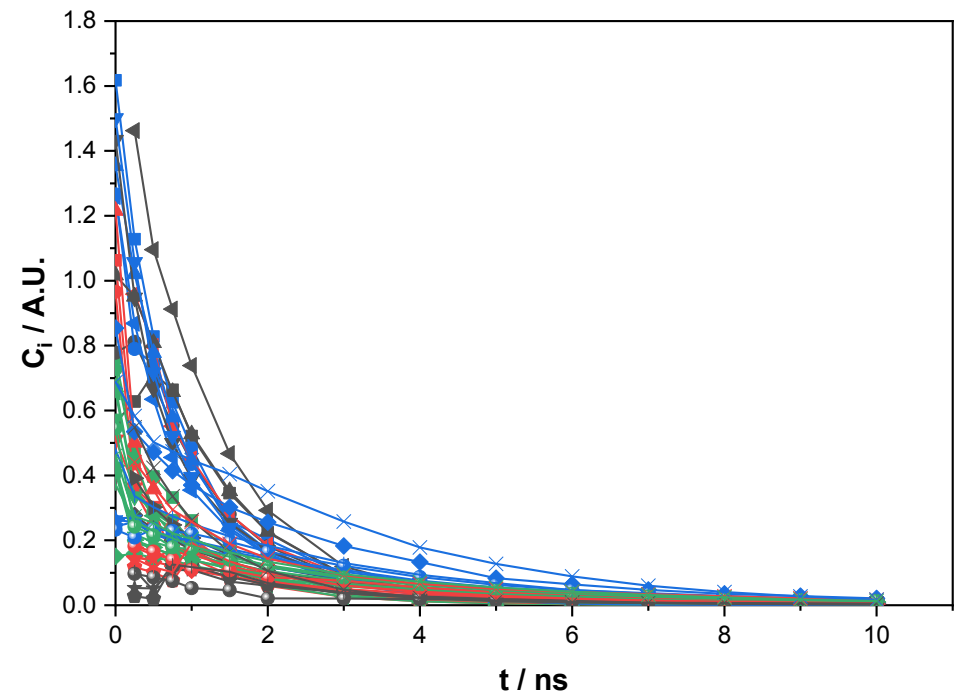

Figure S3: Gaussian models were used to fit TRES spectra for free insulin and insulin with glucose and the peak positions $C_{i}$ were extracted and plotted for $0.33(\boldsymbol{\square}), 22(\bullet), 42(\boldsymbol{\triangleleft}), 68(\boldsymbol{\Delta}), 93(\boldsymbol{\nabla})$, $196(\diamond), 310(\diamond) 379(\star), 458(\bullet), 528(\bullet), 605(\mid)$, and $690(\times)$ hours. Component 1 and 2 for free insulin and insulin with glucose are represented with grey, red, blue, and green, respectively. 


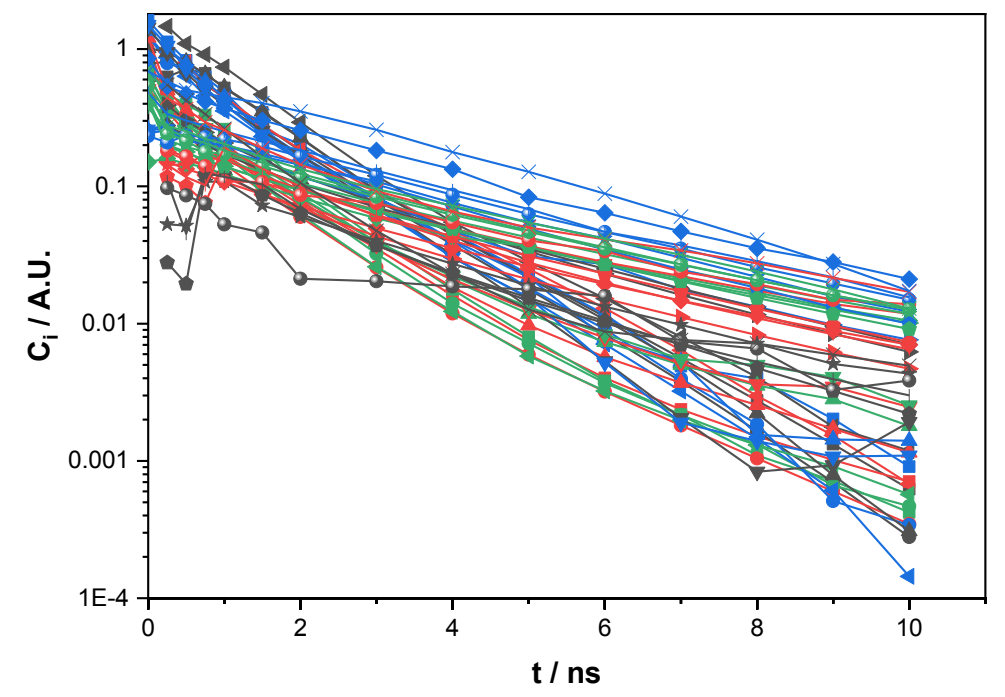

Figure S4: Gaussian models were used to fit TRES spectra for free insulin and insulin with glucose and the peak positions $\boldsymbol{C}_{\boldsymbol{i}}$ were extracted and plotted in $\log$ scale for $0.33(\boldsymbol{\nabla}), 22(\bullet), 42(\bullet), 68$ $(\boldsymbol{\Delta}), 93(\boldsymbol{\nabla}), 196(\diamond), 310(\diamond) 379(\star), 458(\bullet), 528(\bullet), 605(\mid)$, and $690(\times)$ hours. Component 1 and 2 for free insulin and insulin with glucose are represented with grey, red, blue, and green, respectively.
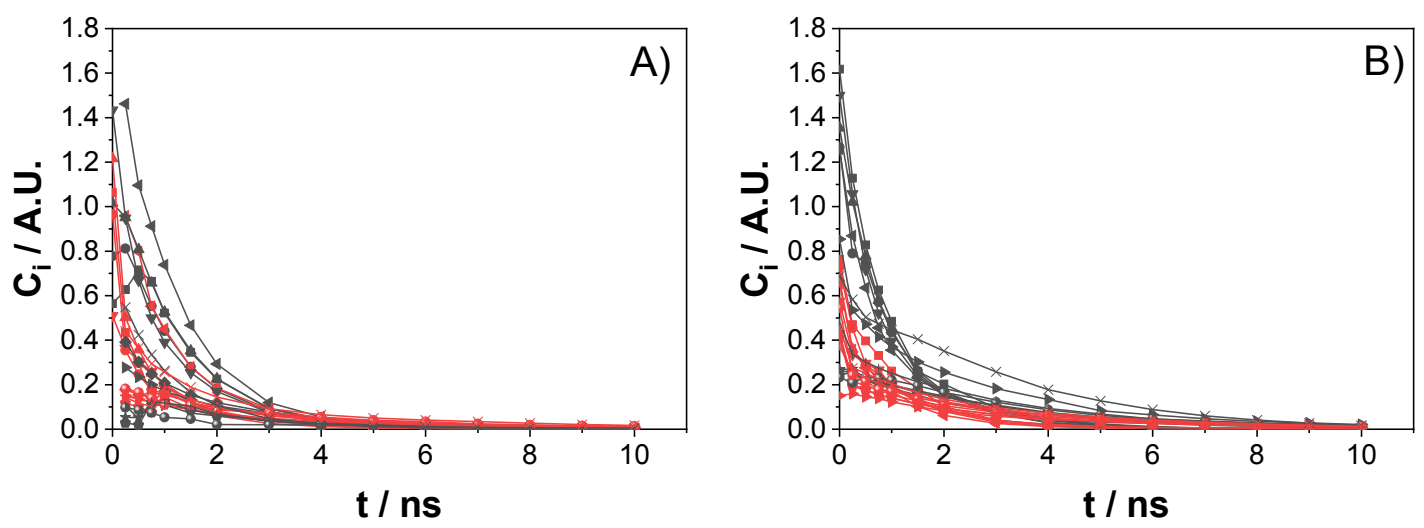

Figure S5: Gaussian models were used to fit TRES spectra for A) free insulin and B) insulin with glucose and the peak positions $C_{i}$ were extracted and plotted for $0.33(\boldsymbol{\bullet}), 22(\bullet), 42(\bullet), 68(\boldsymbol{\Delta}), 93$ $(\nabla), 196(\bullet), 310(\bullet) 379(\star), 458(\bullet), 528(\bullet), 605(\mid)$, and $690(\mathrm{x})$ hours. Components 1 and 2 are represented with grey and red curves, respectively. 


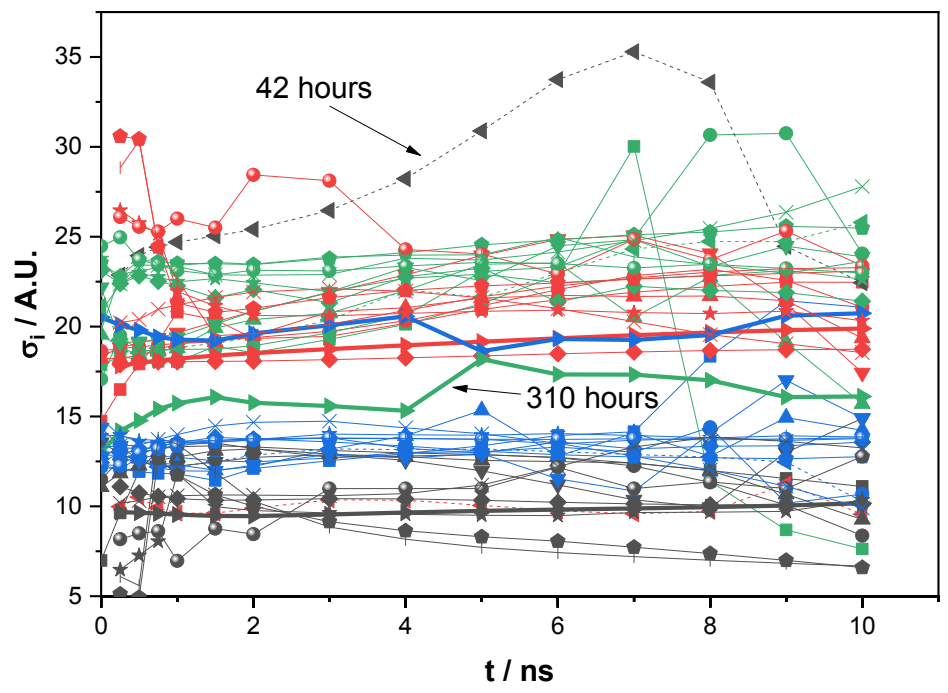

Figure S6: Gaussian models were used to fit TRES spectra for free insulin and insulin with glucose and the peak positions $\sigma_{i}$ were extracted and plotted for $0.33(\boldsymbol{\square}), 22(\bullet), 42(\boldsymbol{\bullet}), 68(\boldsymbol{\Delta}), 93(\boldsymbol{\nabla})$, $196(\diamond), 310(\diamond) 379(\star), 458(\bullet), 528(\bullet), 605(\mid)$, and $690(x)$ hours. Component 1 and 2 for free insulin and insulin with glucose are represented with grey, red, blue, and green, respectively. 